\title{
Efecto de marcar primero en la final de la UEFA Champions League y la UEFA Europa League Effect of scoring first in finals of UEFA Champions League and UEFA Europe League \\ *Francisco Daniel Martínez Martínez, **Higinio González García \\ *Consejería de Educación de la Región de Murcia (España), **Universidad Internacional de La Rioja (España)
}

Resumen. Este estudio tiene como objetivos: i) conocer cómo influye el efecto de marcar primero en el resultado final del partido; ii) comparar el efecto de marcar primero, los goles totales, el periodo de marcar en el partido y el minuto de marcar el primer gol (ganador y perdedor), en las finales de ambas competiciones; $\mathrm{y}$ iii) analizar la evolución que tienen estas variables a lo largo de la historia. La muestra estuvo formada por todas las finales $(N=78)$ disputadas a lo largo de la historia a partido único y en campo neutral de las competiciones europeas UEFA Champions League (UCL) y UEFA Europa League (UEL). La probabilidad de ganar el equipo que marca primero fue del 75.6\%. Cuando se analizó el efecto de marcar primero los resultados no mostraron diferencias estadísticamente significativas entre ambas competiciones, aunque sí se aprecian al analizar la evolución de este efecto a lo largo de la historia en la UCL a favor de las décadas de 1950 y $1960\left(X^{2}=14.15 ; p<.05\right)$. Se concluyó que el efecto de marcar primero en una final continental resulta determinante pues el equipo que se adelanta en el marcador aumenta en gran medida sus probabilidades de conseguir la victoria. En este sentido, los cuerpos técnicos de los clubes que luchan por el título en estas competiciones deben utilizar esta información para trabajar en los entrenamientos y plantear estrategias que les permitan salir al terreno de juego con la intención clara de abrir el marcador.

Palabras Clave: Historia, fútbol, probabilidad, victoria, gol.

\begin{abstract}
The objectives of this research were: i) to study the effect of scoring first on the final result of the match; ii) to compare the effect of scoring first, the total goals, the goal scoring period, and scoring minute of the first goal (winner and loser) in the finals of both competitions; and iii) to analyse the evolution of these variables throughout history. The sample consisted of all the single-match, neutralfield finals $(N=78)$ played over the history of the UEFA Champions League (UCL) and UEFA Europa League (UEL) European competitions. The team scoring first had $75.6 \%$ probabilities of winning. When analysing the effect of scoring first, the results showed no statistically significant differences between the two competitions, although they become significant in regard to the evolution of the scoring first effect throughout the UCL history, in favour of the finals played within 1950s and 1960s $\left(X^{2}=14.15, p<.05\right)$. As a conclusion, the effect of scoring first in a continental final is decisive because any team leading the scoreboard has major chances of winning. In this sense, technical staff at clubs should use this information to work on the training and propose strategies that allow them to go out onto the pitch with the clear intention of scoring first.
\end{abstract}

Keywords: History, football, probability, victory, goal.

\section{Introducción}

El fútbol es un deporte de equipo con un marcador bajo, en el que el 70-75\% de los encuentros finalizan con tres o menos goles (Anderson \& Sally, 2014). De este modo, la escasa puntuación que maneja su marcador se puede considerar una de las razones que justifiquen la importancia de marcar primero (Castellano, 2009). Tanto es así, que abrir el marcador en un partido es un gran predictor del resultado final en el fútbol profesional, obteniendo el equipo que se pone por delante el 65-70\% de las victorias (García-Rubio, Gómez, Lago-Peñas \& Ibáñez, 2015; Lago-Peñas, GómezRuano, Megías-Navarro \& Pollard, 2016; Martínez \& González-García, 2019a; Pic \& Castellano, 2016). El hecho de marcar el primer gol en un encuentro puede ocasionar un momento psicológico positivo que aumente las probabilidades de victoria del conjunto que se adelanta (Sampedro \& Prieto, 2012). En cambio, se produce el efecto adverso en el rival, pues quien encaja el tanto puede pasar por un momento psicológico negativo (Pollard, 2006; Saavedra, Gutiérrez, Fernández \& Sa, 2015), que provoque una crisis en el equipo, reduciendo la confianza de los jugadores, aumentando la desmoralización y disminuyendo la cohesión en el grupo (Bar-Eli, Tenenbaum \& Geister, 2006). En esta línea, Martínez (2018) realiza una revisión sistemática sobre el efecto de marcar primero en el fútbol profesional, e indica que existen es-

Fecha recepción: 14-10-18. Fecha de aceptación: 23-07-19 Francisco Daniel Martínez Martínez fdaniel.martinez@um.es tudios que analizan el efecto que tiene abrir el marcador sobre el resultado final del partido (Lago-Peñas et al., 2016; Michailidis et al., 2013; Sampedro \& Prieto, 2012); otros que lo estudian en función del minuto en el que se ha marcado y la fase de la competición (García-Rubio et al., 2015); también señala aquellos que examinan y cuantifican la interacción entre los goles marcados en los dos periodos del partido (Nevo \& Ritov, 2013); y los que tratan de identificar los indicadores de rendimiento que influyen en el momento en el que el primer gol es marcado (Pratas, Volossovitch \& Carita, 2016). En este sentido, Michailidis et al. (2013) señalan que los equipos de la fase final de la Eurocopa de 2012 que marcaron el primer gol, ganaron aproximadamente el $71 \%$ de sus encuentros, empataron el $22 \%$ y perdieron el $7 \%$; tanto en la fase de grupos como de eliminatoria. Asimismo, Nevo y Ritov (2013) concluyen que abrir el marcador puede acelerar o impedir la consecución del siguiente gol, dependiendo del momento en el que es marcado. En concreto, exponen que a partir del minuto 65 la probabilidad de ganar supera el 50\%.

Por otro lado, la mayoría de investigaciones en este ámbito (García-Rubio et al., 2015; Lago-Peñas et al., 2016; Martínez \& González-García, 2019a; Pratas et al., 2016; Sampedro \& Prieto, 2012) relacionan el efecto de marcar primero con la localización del partido, demostrando que cuando abre el marcador el equipo que juega como local gana alrededor del $73 \%$ de los encuentros, empata el $18 \%$ y pierde un $9 \%$, y cuando marca primero el que juega a domicilio el porcentaje de victorias está próximo al 63\%, el de empates se sitúa alrededor del $20 \%$ y el de derrotas en torno al $17 \%$. No obstante, García-Rubio et al. (2015) y Lago-Peñas et al. (2016) 
destacan que estos resultados dependen, en gran medida, del minuto en el que se anota el primer gol y de la calidad del oponente. Del mismo modo, Pic y Castellano (2017) resaltan la gran influencia que tiene el valor de los goles en las competiciones de ida y vuelta. En este ámbito, Lago-Peñas et al. (2016) dividen los 90 minutos que dura un partido en seis periodos de 15, tres en la primera parte y tres en la segunda, y analizan la importancia que tiene el momento en el que se marca el primer gol. Así, destacan que cuanto más cerca está el final del partido, más decisivo resulta marcar el primer gol. En cambio, cuando se abre el marcador al inicio del encuentro, la influencia en el resultado final es menos importante debido a que el equipo contrario tiene más tiempo para remontar. En esta línea, Martínez y González-García (2018) dividen el tiempo que dura un encuentro de la misma manera, y estudian el momento en el que marcar un gol tiene más influencia sobre la clasificación final. Estos autores señalan que los goles marcados entre los minutos 16 y 45 de la primera parte, son los que más relación tienen con la obtención de una buena posición en la tabla a final de temporada.

Continuando con esta tendencia, Lago-Peñas et al. (2016) y Sampedro y Prieto (2012) calculan una nueva métrica introducida por estos últimos, que denominan ventaja de marcar primero. Esta ventaja se entiende como el número de puntos obtenidos por los equipos que se adelantan en el marcador en función de su condición de local o visitante, expresados como un porcentaje del total de puntos obtenidos por estos equipos (locales o visitantes) en todos los partidos jugados (Lago-Peñas et al., 2016; Michailidis et al., 2013; Pollard, 1986; Sampedro y Prieto, 2012). Así, existe ventaja de marcar primero para los equipos locales y visitantes cuando el porcentaje de puntos que obtiene el club que se adelanta en el marcador es superior al 50\% (los equipos que abren el marcador consiguen más puntos que los que encajan el primer gol), y no se da esta ventaja si se obtiene un valor igual o inferior al 50\% (los equipos que abren el marcador consiguen igual o menor número de puntos que los que reciben el primer gol). Tomando como base esta definición (Lago-Peñas et al., 2016; Sampedro y Prieto, 2012), el efecto de marcar primero para los equipos que juegan la final de la UEFA Champions League (UCL) o UEFA Europa League (UEL) se entiende como el número de victorias obtenidas por los clubes que se adelantan en el marcador, expresados como un porcentaje del total de finales disputadas. De esta forma, existe efecto de marcar primero cuando el porcentaje de victorias que obtienen los clubes que se adelantan en el marcador es superior al 50\% (los equipos que abren el marcador ganan más finales que los que encajan el primer gol), y no se produce este efecto si se obtiene un valor igual o inferior al 50\% (los equipos que abren el marcador consiguen igual o menor número victorias que los que reciben el primer gol) (Lago-Peñas et al., 2016; Michailidis et al., 2013; Pollard, 1986; Sampedro y Prieto, 2012).

Una vez vistos los presupuestos teóricos de este trabajo, y una vez probada la importante influencia que tiene sobre el efecto de marcar primero la localización del partido y la calidad del oponente, el presente trabajo pretende reducir estos «sesgos» analizando este efecto en las finales de los dos campeonatos más importantes de Europa, la UCL y la UEL, debido a que ninguno de los clubes juega en su campo y la calidad de los equipos que luchan por el título se puede considerar similar. Por tanto, este estudio tiene como objetivos: i) conocer cómo influye el efecto de marcar primero en el resultado final del partido; ii) comparar el efecto de marcar primero, los goles totales, el periodo de marcar en el partido y el minuto de marcar el primer gol (ganador y perdedor), en las finales de ambas competiciones; y iii) analizar la evolución que tienen estas variables a lo largo de la historia.

\section{Método}

\section{Participantes}

La muestra estuvo formada por todas las finales $(N=78)$ disputadas a lo largo de la historia a partido único y en campo neutral de las competiciones europeas UCL y UEL, sin contar los partidos terminados con empate a cero que fueron excluidos por la imposibilidad de analizar en ellos el efecto de marcar primero. Respecto a la UCL se analizaron 59 encuentros $(n=59)$, entre los periodos que comprenden desde la temporada 1955-56 hasta 2017-18, que hicieron un total de 63 temporadas, debido a que cuatro de ellas terminaron sin goles, y de la 1973-74, que fue la única final de la máxima competición europea decidida con un partido de desempate, fue excluido el primer partido por no dictaminar a un ganador. En cuanto a la UEL, se analizaron 19 encuentros $(n=19)$, entre los periodos que comprenden desde 1997-98 hasta 20172018, que hicieron un total de 21 temporadas, debido a que dos de ellas terminaron sin goles y todas las anteriores, comprendidas entre 1971-72 y 1996-97, fueron excluidas por ser finales disputadas a doble partido. Los datos se extrajeron de las páginas web oficiales de la UCL (https://es.uefa.com/ uefachampionsleague/index.html) y de la UEL (https:// es.uefa.com/uefaeuropaleague/index.html). Además, se observó que los datos podían ser utilizados con fines de investigación, puesto que tan sólo se prohibía el uso comercial de los mismos.

En la Tabla 1, se muestra un análisis descriptivo de cada competición. Para ello, se ha cuantificado el número de finales terminadas en el tiempo reglamentario, en la prórroga y en la tanda de penaltis, todas ellas expresadas como un porcentaje de todos los encuentros disputados. Como se puede comprobar, los resultados son muy similares entre ambas competiciones, apreciándose un aumento de partidos terminados en prórroga y penaltis conforme pasan las décadas, especialmente en la UEFA Champions League.

\section{Análisis de las variables}

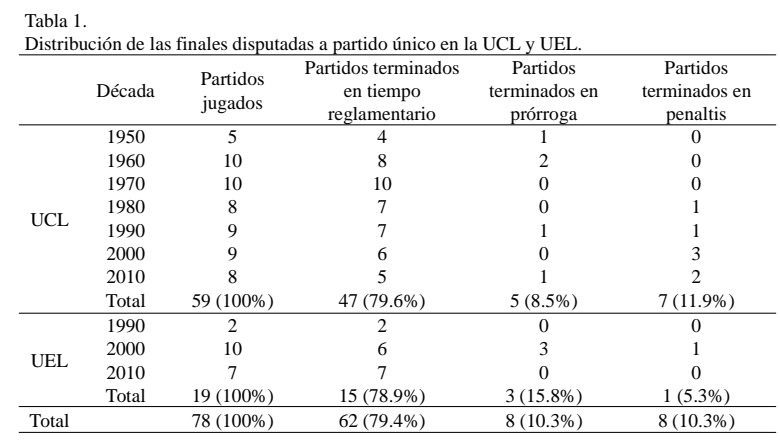

Década: se dividió la muestra de finales disputadas a partido único y en campo neutral en décadas. En la UCL se incluyeron las décadas de 1950, 1960, 1970, 1980, 1990, 2000 y 2010; y en la UEL se incluyeron las décadas 1990, 2000 y 
2010.

Efecto de Marcar Primero: se calculó el porcentaje de victorias que consiguen los equipos que abren el marcador con respecto a todas las finales disputadas.

Goles marcados en función del periodo de juego: se seleccionaron los registros de goles marcados por los equipos en periodos de quince minutos, al igual que en otros estudios previos (Lago-Peñas et al., 2016; Martínez \& GonzálezGarcía, 2018; Martínez \& González-García, 2019a; Martínez \& González-García, 2019b). De esta forma se dividió el partido en 6 periodos de quince minutos, incluyendo el tiempo de descuento de la primera parte en el tercer periodo y el tiempo extra de la segunda parte en el último periodo, resultando de la siguiente forma: 0-15 min. (periodo 1), 16-30 min. (periodo 2), 31-45 min + extra. (periodo 3), 46-60 min. (periodo 4), 61-75 min. (periodo 5), 76-90 min + extra. (periodo 6).

Goles Totales: se calculó el cómputo de goles totales en función de la década y competición (UCL y UEL).

Minuto de Marcar el Primer Gol Ganador: se cuantificó el minuto en que se marcaba el primer gol en cada una de las finales por parte de los equipos ganadores.

Minuto de Marcar el Primer Gol Perdedor: se cuantificó el minuto en que se marcaba el primer gol en cada una de las finales por parte de los equipos perdedores, en caso de que el perdedor no marcara no se computaba este dato en el partido.

\section{Análisis de Datos y Procedimiento}

Para el tratamiento estadístico de los datos se utilizó el programa SPSS 20. Para conocer las características de la muestra, se realizaron los estadísticos descriptivos de media, mínimo, máximo, desviación típica, frecuencia y porcentajes. En los análisis estadísticos se utilizó un error tipo I del 95\% en el empleo de las distintas pruebas estadísticas. Se utilizó la prueba de Komolgorov-Smirnov para conocer si la muestra seguía una distribución normal ( $p>.05)$, como se encontró que la muestra no seguía una distribución normal se utilizaron pruebas no paramétricas. Para calcular las diferencias entre grupos, cuando las variables eran cuantitativas, se utilizó la prueba de Kruskalwallis para muestras independientes. Para calcular las diferencias entre grupos, cuando las variables eran cualitativas, se utilizó la prueba de Chi Cuadrado. Por otro lado, cuando las variables fueron estadísticamente significativas, se calculó el tamaño del efecto a través de la $E t a^{2}$. En este caso, siguiendo a Cohen (1988) se pueden considerar los resultados del tamaño del efecto de la siguiente forma: $c^{2}=.01$ (pequeño), $c^{2}=.06$ (medio), $c^{2}$ $=.14$ (grande).

\section{Resultados}

En la Tabla 2 se expresa en porcentaje el efecto de marcar primero en las finales terminadas en el tiempo reglamentario, en la prórroga y en la tanda de penaltis. Como se puede apreciar, abrir el marcador en las finales de las principales competiciones europeas es sumamente importante para conseguir el título. No obstante, cuando la duración del partido se alarga más allá del tiempo reglamentario, el efecto de marcar primero se reduce.

En la Tabla 3 se realizaron pruebas chi-cuadrado y pruebas Kruskalwallis para conocer las diferencias de medias

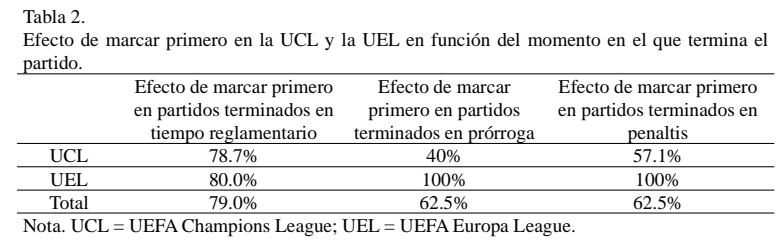

entre las distintas variables de la muestra, los resultados mostraron que existían diferencias estadísticamente significativas $(p<.05)$ en función de la década en la UCL en: el efecto de marcar primero equipo ganador y perdedor, y en el número de goles totales, a favor de las décadas de 1950 y 1960. Además, también se obtuvieron diferencias significativas en los goles en el periodo tres del partido, a favor de la década de 1960 y la década del 2000. Por otro lado, también se encontraron diferencias en el minuto de marcar el primer gol el equipo perdedor $(p<.05)$ a favor de la década de 1950 , mostrando que el equipo perdedor se adelanta en esta década en torno al minuto 27.67. Por otra parte, no se encontraron diferencias significativas ( $p>$.05) en la UEL en función de la década en las distintas variables examinadas (efecto de marcar primero equipo ganador, efecto de marcar primero equipo perdedor, goles totales, goles periodo 1 , goles periodo 2 , goles periodo 3 , goles periodo 4 , goles periodo 5 , goles periodo 6 , minuto marcar primer gol ganador y minuto marcar primer gol perdedor).

En la Tabla 4, cuando se examinaron las diferencias entre la UCL y la UEL juntas en función de la década, se encontraron diferencias significativas en: el efecto de marcar primero equipo ganador y perdedor, y en el número de goles totales, a favor de las décadas de 1950 y 1960. Por otro lado, también se encontraron diferencias en el minuto de marcar el primer gol el equipo perdedor $(p<.05)$ a favor de la década de 1950, mostrando que el equipo perdedor se adelanta en esta década en torno al minuto 27.67. En cambio, cuando se examinaron las diferencias entre ambas competiciones en las distintas variables no se encontraron diferencias significativas ( $p>.05)$.

\section{Discusión}

El presente trabajo tiene como objetivos: i) conocer cómo influye el efecto de marcar primero en el resultado final del partido; ii) comparar el efecto de marcar primero, los goles totales, el periodo de marcar en el partido y el minuto de marcar el primer gol (ganador y perdedor), en las finales de ambas competiciones; y iii) analizar la evolución que tienen estas variables a lo largo de la historia. Teniendo en cuenta los resultados obtenidos, cabe destacar que -a nivel generalel efecto de marcar primero en las finales de la UCL y la UEL se encuentra en el $75.6 \%$, situándose este porcentaje en el $72.9 \%$ para la UCL, y en el $84.2 \%$ para UEL. Esto confirma la inexistencia de diferencias estadísticamente significativas entre ambas competiciones y la importancia que tiene abrir el marcador en este tipo de partidos para conseguir el título. Además, los hallazgos encontrados son similares a los demostrados por otros autores en otro tipo de campeonatos futbolísticos como la fase final de la Eurocopa de 2012, en la que Michailidis et al. (2013) indica que los equipos que marcan el primer gol ganan el 71\% de sus encuentros; o la fase regular de las principales ligas europeas, en las que el porcentaje de victorias del club que se adelanta en el marcador 
Diferencias en función de la década en UEFA Champions League y UEFA Europa League.

Efecto de Marcar Efecto de

Década Primero Equipo Marcar Primero Media Goles Media Goles Media Goles Media Goles Media Goles Media Goles Media Goles Min Marcar Primer Min Marcar Prine

\begin{tabular}{|c|c|c|c|c|c|c|c|c|c|c|c|}
\hline Década & $\begin{array}{c}\text { Ganador } \\
\%\end{array}$ & $\begin{array}{c}\text { Equipo Perdedor } \\
\%\end{array}$ & Totales (SD) & Periodo 1 (SD) & Periodo 2 (SD) & Periodo 3 (SD) & Periodo 4 (SD) & Periodo 5 (SD) & eriodo $6(S D)$ & Gol Ganador $(S D)$ & Gol Perdedor $(S D)$ \\
\hline 1950 & 40 & 60 & $5.20(3.42)$ & $.80(1.30)$ & $.80(1.30)$ & $.20(.44)$ & $.80(.83)$ & $1.80(1.48)$ & $.60(.89)$ & $37(32.85)$ & $27.67(27.79)$ \\
\hline 1960 & 40 & 60 & $4(1.88)$ & .20 (.42) & $.60(.96)$ & $.80(.78)$ & $.60(.51)$ & $1.00(.81)$ & .40 (.51) & 38.70 (19.53) & $44.22(25.60)$ \\
\hline 1970 & 100 & 0 & $1.90(1.19)$ & .20 (.42) & $.30(.48)$ & $.10(.31)$ & $.40(.51)$ & $.40(.51)$ & $.50(.52)$ & 37 (23.44) & $51.00(-)$ \\
\hline 1980 & 87.5 & 12.5 & $1.75(1.16)$ & $.25(.46)$ & $.37(.74)$ & $.25(.46)$ & .25 (.46) & .25 (.46) & $.37(.74)$ & 48.87 (30.05) & $34.00(12.72)$ \\
\hline 1990 & 88.9 & 11.1 & $2.22(1.30)$ & $.22(.44)$ & $.22(.44)$ & $.55(.52)$ & $.22(.66)$ & $.55(.88)$ & $.33(.70)$ & 55.33 (34.47) & $37.33(29.67)$ \\
\hline 2000 & 66.7 & 33.3 & $2.88(1.26)$ & $.55(.72)$ & $.11(.33)$ & $.88(.60)$ & $.44(1.01)$ & $.44(.72)$ & $.44(.88)$ & 38.11 (21.59) & 31.17 (33.65) \\
\hline 2010 & 75 & 25 & 3.62 (1.18) & $.25(.46)$ & $.37(.74)$ & $.25(.46)$ & $.62(.74)$ & $.75(.70)$ & $1.00(.53)$ & $37.62(27.47)$ & 61.38 (22.85) \\
\hline Total & 72.9 & 27.1 & $2.94(1.87)$ & $.32(.60)$ & $.37(.71) 3$ & $.45(.59)$ & $.45(.67)$ & $.63(.84)$ & $.50(.67)$ & $41.94(26.35)$ & $43.44(27.00)$ \\
\hline $\mathrm{X}^{2}(p)$ & $14.15(.028)^{*}$ & $14.15(.028)^{*}$ & $18.91(.00)^{* *}$ & $3.08(.79)$ & $2.92(.81)$ & $13.72(.024)^{*}$ & $6.46(.37)$ & $10.63(.10)$ & $7.83(.13)$ & $2.77(.83)$ & $26.75\left(.00^{* *}\right)$ \\
\hline $\mathrm{Eta}^{2}$ & .19 & .19 & .35 & - & - & .23 & - & - & - & - & - \\
\hline 1990 & 100 & 0 & $3(00)$ & $.50(.70)$ & $.50(.70)$ & $.50(.70)$ & $1(00)$ & $.50(.70)$ & $.00(.00)$ & $15(14.14)$ & $\wedge$ \\
\hline 2000 & 90 & 10 & $4.10(2.02)$ & $.10(.31)$ & $.80(.91)$ & $.70(.82)$ & $1(1.24)$ & .40 (.69) & $.50(.97)$ & 35.90 (19.89) & 35.57 (8.72) \\
\hline 2010 & 71.4 & 28.6 & $3(1.29)$ & $.28(.48)$ & $.42(.53)$ & $.71(.75)$ & $.42(.53)$ & $.71(.95)$ & $.28(.48)$ & $32(18.62)$ & 36.67 (30.53) \\
\hline Total & 84.2 & 15.8 & $3.57(1.70)$ & $.21(.41)$ & $.63(.76)$ & $.68(.74)$ & $.78(.97)$ & $.52(.77)$ & $.36(.76)$ & $32.26(19.10)$ & $35.90(16.07)$ \\
\hline $\mathrm{X}^{2}(p)$ & $1.48(.47)$ & $1.48(.47)$ & $1.72(.42)$ & $1.87(.39)$ & $.60(.73)$ & $.096(.95)$ & $1.41(.49)$ & $.52(.77)$ & $.77(.67)$ & $2.12(.34)$ & $2.94(.22)$ \\
\hline Eta $^{2}$ & - & - & - & (n) & . & - & 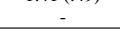 & 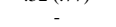 & - & - & . \\
\hline
\end{tabular}

Nota. ${ }^{*} p<.05 ; * * p<.01 ;$ UCL= UEFA Champions League; EL = Europa League; $\wedge$ = No hay casos.

Tabla 4.

Diferencias entre UEFA Champions League y UEFA Europa League a nivel general y en función de la Década.

$$
\text { Efecto de Efecto de }
$$

Marcar Primero Marcar Primero Media Goles Media Goles Media Goles Media Goles Media Goles Media Goles Media Goles Min Marcar PrimerMin Marcar Primer Década Equipo GanadorEquipo Perdedor Totales (SD) Periodo $1(S D)$ Periodo $2(S D)$ Periodo $3(S D)$ Periodo $4(S D)$ Periodo $5(S D)$ Periodo 6 (SD) Gol Ganador (SD) Gol Perdedor (SD)

\begin{tabular}{|c|c|c|c|c|c|c|c|c|c|c|c|c|}
\hline \multirow{9}{*}{$\begin{array}{l}\text { UCL + } \\
\text { UEL } \\
\text { Década }\end{array}$} & 1950 & $\frac{\%}{40}$ & $\frac{\%}{60}$ & $5.20(3.42)$ & $80(1.30)$ & $80(130)$ & $20(44)$ & $.80(.83)$ & $1.80(1.48)$ & $60(89)$ & $37(3285)$ & $27.67(27.79)$ \\
\hline & 1960 & 40 & 60 & $4(1.88)$ & $.20(.42)$ & $.60(.96)$ & . 80 (.78) & $\begin{array}{l}.00(.05) \\
.60(.51)\end{array}$ & $1(.81)$ & . $.40(.51)$ & 38.70 (19.53) & $44.22(25.60)$ \\
\hline & 1970 & 100 & 0 & $1.90(1.19)$ & $.20(.42)$ & $.30(.48)$ & $.10(.31)$ & $.40(.51)$ & $.40(.51)$ & $.50(.52)$ & 37 (23.44) & $51.00(-)$ \\
\hline & 1980 & 87.5 & 12.5 & $1.75(1.16)$ & $.20(.46)$ & $.37(.74)$ & $.25(.46)$ & $.25(.46)$ & $.25(.46)$ & $.37(.74)$ & $48.87(30.05)$ & $34.00(12.72)$ \\
\hline & 1990 & 90.9 & 9.1 & $2.36(1.20)$ & $.25(.46)$ & $.27(.46)$ & $.54(.52)$ & $.36(.67)$ & $.54(.82)$ & $.27(.64)$ & $48(35.17)$ & 37.33 (29.67) \\
\hline & 2000 & 78.9 & 21.1 & $3.52(1.77)$ & $.27(.58)$ & $.47(.77)$ & $.78(.71)$ & $.73(1.14)$ & .42 (.69) & $.47(.90)$ & 36.94 (20.15) & 33.54 (22.69) \\
\hline & 2010 & 73.3 & 26.7 & $3.33(1.23)$ & $.31(.45)$ & . $40(.63)$ & .46 (.63) & $.53(.63)$ & $.73(.79)$ & $.66(.61)$ & 35 (23.12) & $54.64(26.17)$ \\
\hline & Total & 75.6 & 24.4 & $3.10(1.845)$ & $.29(.56)$ & . $38(.69)$ & $.51(.63)$ & $.53(.76)$ & $.64(.83)$ & .47(.69) & $39.87(25.06)$ & $41.64(24.87)$ \\
\hline & $\begin{array}{l}\mathrm{X}^{2}(p) \\
\mathrm{Eta}^{2}\end{array}$ & $\begin{array}{c}15.72(.01)^{*} \\
.18\end{array}$ & $\begin{array}{c}15.72(.01)^{*} \\
.18\end{array}$ & $\begin{array}{c}20.35 \\
(.00)^{* *}\end{array}$ & $1.31(.97)$ & 1.09 (.98) & $12.29(.056)$ & $3.51(.74)$ & $11.52(.07)$ & $\begin{array}{l}4.81(.56) \\
-\end{array}$ & $1.89(.92)$ & $\begin{array}{c}21.69(.001)^{* *} \\
.23\end{array}$ \\
\hline \multirow{4}{*}{$\begin{array}{c}\text { Diferenci } \\
\text { as entre } \\
\text { UCL y } \\
\text { UEL }\end{array}$} & UCL & 72.9 & 27.1 & $2.94(1.87)$ & . $32(.60)$ & $.37(.71)$ & $.45(.59)$ & $.45(.67)$ & $67(85)$ & $.50(67)$ & $41.94(26.35)$ & $43.44(27.00)$ \\
\hline & UEI & 842 & 158 & $357(170)$ & $21(41)$ & $63(76)$ & $68(74)$ & $78(97)$ & $52(77)$ & $36(76)$ & $3226(1910)$ & $35.90(16.07)$ \\
\hline & $\mathrm{X}^{2}(p)$ & $1.01(.31)$ & $1.01(.31)$ & $2.81(.09)$ & $.33(.56)$ & $2.72(.09)$ & $1.35(.24)$ & $1.83(.17)$ & .49(.48) & $1.20(.27)$ & $1.68(.19)$ & $.16(.68)$ \\
\hline & $\mathrm{Eta}^{2}$ & - & - & - & - & - & - & - & - & - & - & - \\
\hline
\end{tabular}

se establece entre el $63 \%$ y el $73 \%$, dependiendo de si el conjunto que marca primero es local o visitante (Lago-Peñas et al., 2016; Martínez \& González-García, 2019a; Pratas et al., 2016; Sampedro \& Prieto, 2012). En cambio, se alejan más de los encontrados por García-Rubio et al. (2015), quienes en la fase de grupos y de eliminatorias en la UEFA Champions League, señalan un porcentaje alrededor del $60 \%$, en función de la localización del partido y la calidad del oponente. Además, el efecto de marcar primero en función del momento en el que termina el partido también está próximo a estos porcentajes, siendo del $79 \%$ en las finales acabadas en el tiempo reglamentario, y del 62.5\% en las que concluyen en la prórroga y penaltis. Desde esta óptica, sería interesante seguir investigando cómo varía este efecto en función del momento en el que termina el encuentro debido a que no hay estudios con los que poder contrastar los resultados que aquí se presentan.

Por otra parte, si se analiza la evolución que ha tenido el efecto de marcar primero a lo largo de la historia, se puede comprobar cómo en las dos primeras décadas de las UCL no existía este efecto debido a que se situaba por debajo del 50\% (Lago-Peñas et al., 2016; Sampedro y Prieto, 2012). En cambio, a partir de 1970 ha resultado determinante para la consecución del título. De hecho, durante esa década en concreto todos los equipos que se adelantaron en el marcador consiguieron la victoria. Como demuestran los resultados, esto puede ser debido a que en los inicios de la UCL el número de goles que se marcaban en las finales era significativamente más alto que en los años venideros (Tuðbay, 2018) y, por tanto, abrir el marcador no resultaba decisivo. No obstante, se debería seguir investigando en esta línea puesto que son resultados que se aportan a la comunidad científica y no existen trabajos con los que com- pararlos.

En cuanto a la evolución que ha tenido el efecto de marcar primero a lo largo de la historia de la UEL, no existen diferencias estadísticamente significativas en ninguna de las variables estudiadas. Esto puede ser debido a que las finales disputadas a partido único y en campo neutral en esta competición, comenzaron a jugarse a partir de la temporada 199798 y, como consecuencia, la forma de jugar de los equipos durante este tiempo no ha sufrido grandes variaciones y, además, el número de encuentros analizados es reducido. A pesar de ello, estos datos no se han podido contrastar con otros estudios, por lo que se recomienda continuar con esta línea de investigación para poder extender estos resultados a otras competiciones.

En otro ámbito, la media de goles que se marcan en las finales de las dos competiciones de fútbol más importantes del continente europeo es de 3.10 por partido, estableciéndose en 2.94 goles en la UCL y 3.57 en la UEL. Estos hallazgos están por encima de los encontrados por Anderson \& Sally (2014), quienes indican que el número de goles anotados por partido en las principales ligas europeas es de 2.66. A pesar de ello, se corrobora que el marcador que se maneja en este deporte es bajo, tal y como afirma Castellano (2009). Continuando con esta tendencia, si se analiza por periodos la media de goles de la UCL a lo largo de la historia, se ve la existencia de diferencias estadísticamente significativas en el periodo tres, a favor de las décadas de 1960 y 2000. Esto puede ser debido a que ha existido una gran variabilidad de goles anotados durante estas décadas en esos minutos del partido. No obstante, resulta curioso que el mayor número de goles que se marcan en esta competición es en el periodo cinco (.63), y más relevante aún se puede considerar que en las finales de la última década el mayor número de goles se 
haya conseguido en los dos últimos periodos del partido (.75 y 1.00 respectivamente). Además, en el caso de la UEL, el periodo en el que se marcan más goles es el cuarto (.78). Estos resultados están en la línea de los que argumentan autores como Nevo y Ritov (2013), quienes exponen que abrir el marcador a partir del minuto 65 aumenta la probabilidad de ganar por encima del 50\%, y de los que señalan LagoPeñas et al. (2016), quienes destacan que cuanto más cerca está el final del partido, más decisivo resulta marcar el primer gol.

No obstante, en la UCL el ganador suele abrir el marcador en el minuto 42 de partido y en la UEL en el 32. A pesar de ello, no existen diferencias estadísticamente significativas entre ambas competiciones. Estos resultados están en consonancia con los aportados por Martínez y González-García (2018), quienes señalan que los goles marcados entre los minutos 16 y 45 del encuentro son los que más influencia tienen sobre la clasificación final.

En cuanto a las limitaciones, cabe resaltar el tamaño reducido de la muestra, por lo que se debe tener cautela a la hora de comparar estos resultados con otros estudios que analizan variables similares en el fútbol profesional, o con campeonatos futbolísticos de menor nivel. En esta línea, también resulta complejo comparar el efecto de marcar primero en las finales a partido único y en campo neutral de la UCL y la UEL debido a la diferencia del tamaño de la muestra entre ambas competiciones. Por último, la inexistencia de trabajos que hubieran analizado estas variables con una muestra similar ha aumentado la complejidad a la hora de contrastar los resultados.

Como propuesta futura de investigación, además de las ya comentadas, sería interesante ampliar la muestra a finales de campeonatos nacionales que presentan las mismas características que las analizadas en este trabajo, como por ejemplo la Copa del Rey en España, o incluso a las eliminatorias directas de la fase final de la Copa Mundial de la FIFA.

A modo de conclusiones, este trabajo demuestra la enorme importancia que tiene el efecto de marcar primero en el resultado de una final continental, tanto de la UEFA Champions League como de la UEFA Europa League, pues el equipo que se adelanta en el marcador aumenta en gran medida sus probabilidades de conseguir la victoria. Además, si el partido termina en el tiempo reglamentario, sin necesidad de disputar prórroga o penaltis, las posibilidades son aún mayores. Esta tendencia se mantiene a lo largo de la historia, por lo que los cuerpos técnicos de los clubes que luchan por el título en estas competiciones deben utilizar esta información para trabajar en los entrenamientos y plantear estrategias que les permitan salir al terreno de juego con la intención clara de abrir el marcador.

\section{Agradecimientos}

González-García quiere agradecer a la Universidad Internacional de La Rioja (UNIR) la financiación prestada para esta publicación.

\section{Referencias}

Anderson, C., \& Sally, D. (2014). The Numbers Game: Why Everything You Know about Football Is Wrong. New York: Penguin Books.

Bar-Eli, M., Tenenbaum, G., \& Geister, S. (2006). Consequences of players' dismissal in professional soccer: A crisis-related analysis of group-size effects. Journal of Sports Sciences, 24(10), 10831094. doi:10.1080/02640410500432599

Castellano, J. (2009). Conocer el pasado del fútbol para cambiar su futuro. Acción motriz, 2, 1-19.

Cohen, J. (1988). Statistical Power Analysis for the Behavioral Sciences. New York: Academic Press.

Courneya, K. S. (1990). Importance of game location and scoring first in college baseball. Perceptual and Motor Skills, 71(2), 624-626. doi:10.2466/pms.1990.71.2.624

García-Rubio, J., Gómez, M. Á., Lago-Peñas, C., \& Ibáñez, J. S. (2015). Effect of match venue, scoring first and quality of opposition on match outcome in the UEFA Champions League. International Journal of Performance Analysis in Sport, 15(2), 527-539. doi:10.1080/24748668.2015.11868811

Jones, B. M. (2009). Scoring first and home advantage in the NHL. International Journal of Performance Analysis in Sport, 9(3), 320-331. doi:10.1080/24748668.2009.11868489

Lago-Peñas, C., Gómez-Ruano, M., Megías-Navarro, D., \& Pollard, R. (2016). Home advantage in football: Examining the effect of scoring first on match outcome in the five major European leagues. International Journal of Performance Analysis in Sport, 16(2), 411421. doi:10.1080/24748668.2016.11868897

Martínez, F.D. (2018). Efecto de marcar primero en el fútbol profesional: Revisión Sistemática. Revista Actividad Físicay Deporte: Ciencia y Profesión, 28(1), 9-19.

Martínez, F.D., \& González-García (2018). Análisis de los goles en el fútbol europeo y momento en el que influyen más sobre la clasificación final. E-balonmano.com: Revista de Ciencias del Deporte, 14(2), 89-98.

Martínez, F.D., \& González-García (2019a). Efecto de marcar primero y la localización del partido en las principales ligas del fútbol europeo. Retos, 35(1), 242-245.

Martínez, F.D. , \& González-García (2019b). Effect of scoring first and match period in: Football World Cup and UEFA Euro. European Journal of Human movement, 42(1), 123-137.

Michailidis, Y., Michailidis, C., \& Primpa, E. (2013). Analysis of goals scored in European Championship 2012. Journal of Human Sport \& Exercise, 8(2), 367-375. doi:10.4100/jhse.2012.82.05

Nevo, D., \& Ritov, Y.A. (2013). Around the goal: examining the effect of the first goal on the second goal in soccer using survival analysis methods. Journal of Quantitative Analysis in Sports, 9(2), 165177. doi:10.1515/jqas-2012-0004

Pic, M., \& Castellano, J. (2016). Efecto de la localización del partido en eliminatorias de ida y vuelta de la UEFAChampions League. Revista Internacional de Ciencias del Deporte, 44(12), 149-163.

Pic, M., \& Castellano, J. (2017). Influence of match location in the Spanish Copa del Rey. Retos, 31, 202-206.

Pollard, R. (1986). Home advantage in soccer: A retrospective analysis. Journal of sports sciences, 4(3), 237-248. doi:10.1080/ 02640418608732122

Pollard, R. (2006). Home advantage in soccer: variations in its magnitude and a literature review of the inter-related factors associated with its existence. Journal of Sport Behavior, 29, 169-189.

Pratas, J. M., Volossovitch, A., \& Carita, A. I. (2016). The effect of performance indicators on the time the first goal is scored in football matches. International Journal of Performance Analysis in Sport, 16(1), 347-354. doi:10.1080/24748668.2016.11868891

Saavedra, M., Gutiérrez, O., Fernández, J. J., \& Sa, P.(2015). Measuring home advantage in Spanish football (1928-2011). Revista Internacional de Medicina y Ciencias de la Actividad Física y el Deporte, 15(57), 181-194.

Sampedro, J., \& Prieto, J. (2012). El efecto de marcar primero y la ventaja de jugar en casa en la liga de fútbol y en la liga de fútbol sala de España. Revista de psicología del deporte, 21(2), 301-308.

Tuðbay, I. (2018). An analysis of the impact of the change in scoring system on home field advantage soccer leagues. Universal Journal of Educational Research, 6(5), 823-829. doi:10.13189/ ujer.2018.060502 